University of Nebraska - Lincoln

DigitalCommons@University of Nebraska - Lincoln

Faculty Publications, Department of Child, Youth, and Family Studies

Child, Youth, and Family Studies, Department of

June 2004

\title{
Addressing the mental health needs of the rural underserved: Findings from a multiple case study of a behavioral telehealth project
}

\author{
Richard Bischoff \\ University of Nebraska-Lincoln, rbischoff2@unl.edu \\ Cody S. Hollist \\ University of Nebraska Lincoln, chollist2@unl.edu \\ Craig W. Smith \\ University of Nebraska-Lincoln, csmith4@unl.edu \\ Paul Flack \\ Loa, UT
}

Follow this and additional works at: https://digitalcommons.unl.edu/famconfacpub

Part of the Family, Life Course, and Society Commons

Bischoff, Richard; Hollist, Cody S.; Smith, Craig W.; and Flack, Paul, "Addressing the mental health needs of the rural underserved: Findings from a multiple case study of a behavioral telehealth project" (2004). Faculty Publications, Department of Child, Youth, and Family Studies. 44.

https://digitalcommons.unl.edu/famconfacpub/44

This Article is brought to you for free and open access by the Child, Youth, and Family Studies, Department of at DigitalCommons@University of Nebraska - Lincoln. It has been accepted for inclusion in Faculty Publications, Department of Child, Youth, and Family Studies by an authorized administrator of DigitalCommons@University of Nebraska - Lincoln. 


\title{
Addressing the Mental Health Needs of the Rural Underserved: Findings from a Multiple Case Study of a Behavioral Telehealth Project
}

\author{
Richard J. Bischoff \\ Cody S. Hollist \\ Craig W. Smith \\ Paul Flack
}

ABSTRACT: Behavioral telehealth is a reasonable solution to the accessibility to mental health care problem that exists in many rural communities. This paper reports the results of a multiple case study of a behavioral telehealth program administered through a marriage and family therapy training program. The results suggest that mental health services can be effectively delivered using existing distance education technology to underserved rural populations. Rural communities have unique barriers to accessing mental health care, some of which can be overcome through the distance delivery of services and some of which cannot. In order to effectively deliver treatment, accommodations to the technology must be made by both therapist and client.

KEY WORDS: behavioral-telehealth, telecounseling, rural mental health, rural underserved

Rural communities throughout the United States have experienced substantial increases in the incidence of psychopathology and relationship distress (Hoyt, Conger, Valde, \& Weihs, 1997). The

Richard J. Bischoff, PhD, and Craig W. Smith, PhD, Marriage and Family Therapy Program, University of Nebraska-Lincoln, PO Box 830801, Lincoln, NE 685830801 (rbischof@unlnotes.unl.edu). Cody Hollist, MS, doctoral candidate, Brigham Young University. Paul Flack, MS, Loa, UT. Address correspondence to the first author. 
negative consequences of leaving these problems untreated are well known to mental health workers and have been documented in the literature. They include poor health outcomes, suicide, divorce, substance abuse, child neglect and abuse, and youth delinquency (Blumenthal \& Kagen, 2002; Craft \& Staudt, 1991; Nebraska Health and Human Services, 2002; Johnson \& Booth, 1990; Kim, Conger, Lorenz, \& Elder, 2001). Early treatment reduces the far-reaching consequences of psychopathology and relationship distress (Amundson, 2001). Because rural communities are often without accessible mental health care, many rural residents are particularly at risk for the negative consequences of mental health problems (e.g., Chalifoux, Neese, Buckwlater, Litwak, \& Abraham, 1996; Roberts, Battaglia, Smithpeter, \& Epstein, 1999).

In Nebraska, 67 of 93 counties $(72 \%)$ are considered Mental Health Professional Shortage Areas, meaning that the number of mental-health professionals practicing in those counties is insufficient to meet the need for care. Most distressing is the fact that $22(24 \%)$ counties have no mental health services at all (Nebraska Health and Human Services, 2002). Consequently, many rural residents with mental-health problems must travel prohibitive distances to access care, only to be put on lengthy wait-lists before receiving treatment in overburdened mental-health clinics. Given such circumstances, many who require care choose not to seek it. The emotional and community costs of such inaccessibility to care represent a significant and pervasive problem throughout rural America. There is a critical need to overcome this increasingly important access to care problem.

\section{TELECOMMUNICATIONS AS A MEDIUM FOR DELIVERING MENTAL HEALTH SERVICES}

The use of telecommunications technologies is an innovative approach that has been used successfully to improve accessibility of specialty health care to rural and urban underserved populations (e.g., Chavez, 1997; Nickelson, 1998). When psychiatric and other mental health care services are delivered using telecommunications it is generally referred to in the scholarly literature as behavioral telehealth (BTH), although many other terms have also been used (e.g., telepsychiatry, distance counseling/therapy, behavioral ehealth, etherapy). The initial successes of BTH programs (Miller, 2001) suggest that use of telecommunications is a promising solution to the inaccessibility of mental-health services in rural commu- nities where more traditional treatment options do not exist (Nickelson, 1998; Rohland, Saleh, Rohrer, \& Romitti, 2000).

\section{THE BEHAVIORAL TELEHEALTH PROGRAM (BTH) AT THE UNIVERSITY OF NEBRASKA-LINCOLN}

In the Spring of 1999, faculty associated with the marriage and family therapy (MFT) program at the University of Nebraska-Lincoln began discussing the possibility of developing a BTH program that would address the needs of the rural underserved. A pilot BTH program was initiated in January 2001 using the state-wide educational telecommunications infrastructure. End-user sites were high schools in two contiguous rural counties in the Sandhills region of the state. These high schools were already equipped with state-ofthe-art telecommunications technology used in resource sharing and course delivery. Services were delivered at no cost through the Family Resource Center (FRC) - the on-campus marriage and family therapy training clinic.

Through this program, mental health services were delivered through a combination of satellite and DSN (Digital Signal Network) cable. This high speed telecommunications option provided excellent audio and video quality. The video cameras in the FRC and end-user facilities were set up so that both therapist and client could see one another from head to toe and multiple people could be observed at one time, thus accommodating family treatment. Because it is a proprietary system, the risk of breaches of confidential ity were negligible.

In initiating the BTH program, the faculty anticipated the need to address the following in order to successfully administer treatment:

- Because the client and therapist would not be physically proximal, it was anticipated that some accommodations in the therapy would need to occur in order to compensate for any loss in the therapeutic alliance associated with physical proximity. However, it was not clear what these accommodations would need to be.

- Because the telecommunication system's primary function was delivering distance education to rural communities scheduling therapy was restricted to availability of both the system and the end-user facilities.

- Because of the data transmission, a slight delay in the signal was experienced. It was anticipated that this delay would be 
disruptive to the pacing as compared to normal face-to-face communication.

- Although the data transmission was secure, clients had to receive services at the rural county high school where the distance education equipment was located.

- Because the high school is a public place, clients could be seen coming and going from treatment. This was exacerbated by the fact that the high school facilities had to be scheduled by the principal or a member of the school staff.

- The medium of treatment delivery and the confluence of other disadvantages could make it difficult or impossible to accommodate all client problems. For example, scheduling limitations and physical distance could have made this mode of delivery inappropriate for dealing with severe pathology and other problems that could potentially require crisis intervention. It was believed by the program coordinators that the treatment modality would be most appropriate for moderately to mildly distressed individuals and that alternatives would need to be established to accommodate severe distress.

Several noteworthy strategies were used to address these potential problems. First, MFT faculty provided training for school personnel on the importance of confidentiality and client privacy. Second, a Consent to Treatment form was developed that, in addition to informing clients of typical legal and ethical issues of therapy, informed them of each of the disadvantages to this mode of treatment delivery and the limitations of BTH treatment. Third, all scheduling was handled by the FRC staff in an effort to reduce the contact between client and school personnel. Fourth, MFT faculty developed working relationships with therapists who were providing services at the nearest mental health facility. They were enlisted as consultants and as referral sources in the event that problem severity exceeded the capacity of the medium of treatment delivery.

It was determined that a study of this innovative treatment delivery strategy could accomplish two primary purposes. First, a disciplined inquiry about the experience of treatment through BTH could improve the services provided through the program. Given that no one involved in the program had previously provided treatment using this medium, it was important that rigor be employed in the assessment process in order to match client with treatment needs and to improve the program most effectively. In order to maintain this rigor, the study design was patterned after that of action research (Moon \& Trepper, 1996). Second, the study was designed so that the results would have broader applicability to others within the field. While BTH is not new, little is known about the process, structure, or issues needing to be addressed in order for BTH to be successful (Miller, 2001). It was reasoned that an exploratory study of the experience of BTH would advance the field.

\section{METHODS}

A formal multiple case study design (Moon \& Trepper, 1996 Yin, 1989) was used to accomplish the research goals. These goals were to 1) identify the therapist, client, and technology factors affecting the delivery of services; 2) articulate the characteristics of service delivery through this telecommunications strategy; and 3) identify a list of treatment recommendations.

\section{Case Selection and Description}

To accomplish the purposes of the research, a purposeful case selection strategy (Yin, 1989) was chosen. Before initiating any treatments, it was decided that the first case treated through the behavioral telehealth program would be selected for study. Shortly after the study of this treatment was initiated, it was decided that the study of additional cases would be beneficial, so the next two referrals to the program were also selected for inclusion. As will be described below, the second and third cases differed sufficiently from the first to provide a broader view of the BTH program.

It was assumed that the differences from traditional face-toface treatments would be pronounced and that using the first instances of treatment provision would provide the richest perspective on the issues. Therapists, supervisors, and researchers were all free of previous experience that could unwittingly bias their experience. This resulted in good data for study because mistakes were common and learning from mistakes to improve the program was everyone's priority.

Two therapists provided treatment. Both therapists were in their final year of their master's degree clinical training program in marriage and family therapy and were selected because of their special interest in rural mental health and their notable skill in joining and other therapeutic processes. Both therapists had been residents of rural communities throughout their childhood. One 
therapist provided treatment only to Case A. On account of this therapist's graduation, this case was transferred to the other therapist after 17 sessions. The second therapist was providing treatment for cases $\mathrm{A}$ and $\mathrm{B}$ at the time of data collection and had recently discontinued sessions with Case $\mathrm{C}$ as a result of achieving treatment goals.

Case A consisted of a 17-year-old adolescent Caucasian male (Client A) referred to treatment by high school personnel. Although both parents agreed to participate in treatment, the father did not attend any sessions. The basis for the referral as described by the school counselor provides a glimpse into the culture of the rural community and the perceived need for services:

We identified [him as] ... falling through the cracks and we're a small school and you're not supposed to fall through the cracks here. We pride ourselves on not having dropouts, and we pride ourselves on being able to get every one of our students through school and to get every one of them to at least care enough to pass and that wasn't happening with [him]. We were seeing that he was really apathetic, he expressed real hate for school and seemed to be kind of depressed, so we just thought that he needed some help. And when you came to us and said that this might be a possibility ... he was the one that came to mind immediately ... maybe a professional counselor could help him.

Case B was a Caucasian woman in her mid-40s (Client B) who requested treatment after hearing a presentation about the availability of BTH services. This woman requested marital therapy due to dissatisfaction in her marriage. Despite efforts by both client and therapist, her husband refused to participate in treatment.

Case C was a Caucasian woman in her mid-40s (Client C) who learned of the availability of treatment through client B. This woman also requested marital therapy for similar reasons. Similar to Case B, her husband refused participation.

\section{Investigators}

The principal investigators were a faculty member associated with the MFT program and a student in his final year of the clinical training program. The faculty member was chiefly responsible for establishing the BTH program and so had been involved in the de- tails of the selection of the telecommunications strategy used, the review of the literature that informed the initial BTH protocol, and the coordination with and training of end-user site personnel. The faculty member did not have experience providing therapy through BTH and did not supervise any of the BTH cases. The student did not have experience providing therapy through BTH. At the outset of the program, both investigators hypothesized that the physical separation would be a significant barrier that would require accommodations to the normal provision of therapy in order to fa cilitate success. Another investigator was added as the data analysis progressed as a model commentator to aid in triangulation (Denzin, 1978).

\section{Triangulation}

Several methods were implemented to achieve triangulation (Denzin, 1978). Some noteworthy methods that may not be apparent elsewhere are the following. A) As the data analysis progressed a model commentator was added. The role of this person was to read the results as they emerged from the analysis comparing them with the transcripts. The purpose was to ensure that the most important themes were being represented in the results. B) The reconstructions of the data were reviewed by one of the therapists who provided therapy through the telecommunications system. This is a process known as member checking (Yin, 1989). C) Three client systems were sources of data for the study. Interviews were conducted in such a way that results from one interview informed the questioning of subsequent interviews. So, as data analysis was occurring, investigators asked for clarification and confirmation of the emerging results with other participants.

\section{Data Collection and Analytic Procedures}

All BTH sessions were videotaped and observed. Observations were recorded, shared, and discussed among the investigators. As a result, questions were generated to assess the therapist and client experience of this method of treatment delivery. Special note was made of situations or conversations in therapy that appeared unique to this mode of treatment delivery. An interview was conducted individually with the therapists and with each client. Interviews were also conducted with each person involved in the treat ment delivery for the identified cases (i.e. superintendent, school secretary, therapist's supervisor, and so on). All interviews were 
audiotaped and transcribed. Analysis was conducted through open and axial coding (Strauss \& Corbin, 1990) of the transcribed statements made by participants. Observations of videotaped sessions were used to inform this coding but were not coded. Using open coding, participant statements were analyzed and coded to represent the meaning of the statement. Using a constant comparison method (Strauss \& Corbin, 1990), statements were compared and coded in the same way when the meanings of the statements appeared similar. In an effort to capture the richness and diversity of experiences, care was taken during this phase of the data analysis to emphasize the diversity of statements made without prematurely grouping statements. Axial coding was then used to compare categories of data both within cases and across cases in an effort to reduce the number of categories and to develop an elegant and parsimonious description of the experience of participation in a BTH program.

\section{RESULTS}

Based on analysis of the data, two key assertions can be made. First, the context and culture of rural communities inhibits access to mental health care. This assertion is informed by participant descriptions of the challenges of accessing mental health care regardless of the treatment delivery medium. Second, technology mediated therapeutic conversations are different than traditional face-to-face therapeutic conversations. This assertion is informed by participant descriptions of what it is like to receive and provide treatment using the identified telecommunications medium.

\section{Assertion 1: Residing in Rural Communities Inhibits Access to Mental Health Care}

The inaccessibility of mental health care was a concern expressed by each of the rural participants-including the 17-year-old male client. In most cases, comments reflecting participant concerns about inaccessibility of services were made without prompting from the interviewer. Participants explained that barriers to accessing traditional mental health care were often insurmountable given the demands of work, home, and community life. While the BTH program eliminated some barriers, other barriers persisted due to what participants perceived as the nature of living in rural communities. Participant descriptions of the barriers to accessing mental health care concerned the distance of rural residents from traditional clin- ical services and the perceived difficulty of keeping knowledge of receiving therapy out of the public domain.

The distance to traditional mental health services and associated problems with scheduling. Rural participants explained that traditional therapy, where therapist and clients meet face-to-face, do not meet the time demands of most rural residents. Client B, who had previously received traditional mental health treatment, explained that rural residents-some of whom live two to three hours away from the nearest therapeutic services-may be forced to take up to an entire day off work in order to accommodate a session. Thus, the financial burden of therapy is exacerbated by the many hours of missed work as well as travel costs. This is especially difficult for farmers, ranchers, and those who are paid an hourly wage. Delivering mental health services to rural communities through BTH programs can overcome these barriers by making services more convenient and less costly.

Client B summarizes these barriers by saying:

[The nearest counselor is] 120 miles, one way . . . so that's a whole day invested in getting there, having an appointment, and getting back . . you can't just take an hour off of work or off of whatever your day is and go to your counseling appointment. It's a day-long commitment . . . [If] something comes up . . . under normal circumstances you would be able to accommodate both those things. But, if one other thing comes up during that day, then I have to cancel and reschedule that appointment. So logistically, it's hard. Then, [there is the financial hurdle associated with traveling]. Last year, when gasoline prices were high, it cost me an extra $\$ 40$ to get to my appointment and back, just in the gasoline ... So, for somebody ... who doesn't get paid if [they don't] work . . . [it would be] just an extra dis-incentive . . . because it is a whole day [they have] to take off.

The distance from treatment is not just a time, cost, and scheduling barrier. It may also increase the perceived stigma of therapy. Another female participant explained that given the nature of small town communities, taking off work for a day to go to therapy would create suspicion in people about the severity of symptomsthereby increasing the stigma of needing what would seem to be a full day of therapy. Having services offered in the community can potentially reduce this stigma. She said: 
You don't have to make the big announcement that you're going to be gone [for an extended period] to go do something and everybody's wondering why. You know, you're gone for an hour and that's it. It's done ... I really think that this would cause less commotion than if somebody found out they were going [out of town] for counseling.

Participants universally expressed a perception that residents of rural communities are reluctant to go to therapy because of a stigma associated with needing therapy to solve problems. Each of the adult rural participants speculated that rural residents are more reluctant to go to therapy than are urban residents. For example, a high school secretary explained:

Rural people probably go to a therapist after they've pursued every other avenue of solving their situation. I think rural people kind of have the mind-set that they ought to be able to take care of things, when in a more urban area, I think people think, 'Hey, I got a problem. I should go talk with somebody about it.' It would be kind of like going to a medical doctor. They think of it similarly. But, I think that we are a little bit behind the times in that getting therapy is really something that most normal people don't need.

The perception that everyone knows everyone else's business. The stigma of counseling is fueled by the belief that everyone in a rural community knows everyone else's business. The school counselor with Case A explained, "Sometimes people don't go to get help because of that stigma that everyone will know."

One of the hallmarks of ethical psychotherapy practice is confidentiality. Therapists should keep confidential both the content of the sessions and the fact that someone is receiving therapy. While the telecommunications system allowed for confidential communications during the BTH sessions, the location of service delivery limited the ability to keep confidential the fact that clients were attending therapy. Respondents indicated that because the high school was a center of community activity, they at times felt conspicuous receiving help. Client B explained, "there's no such thing as being anonymous in a community this size . . . You just have to be real careful. And with the counseling set up ... there's no way you can be careful." But, they treated this conspicuousness as a fact-of-life when living in a rural community. They believed that others learning of their therapy was inevitable, whether they had to travel to get it or whether it was brought to them through telecommunications at the high school. What was more important to them than public knowledge of their participation in therapy was that the conversations with their therapist were confidential. A school counselor working with Case A summarized the rural dynamics associated with confidentiality:

I can tell you that most of what people know about [the client] probably comes from [the client] or [his family] ...

We have confidentiality, but it's really hard to be confidential when ... everyone in this building probably knows that you are here and I am in the office with you. And it's probably like, who is that? What do they want? . . . The kids tell [each other] where they're going or sometimes they send out spies to say they need to go to the bathroom and really they're checking to see what's going on ... It's a small community and everybody cares about everybody. So, you know, I think we're very confidential and very professional ... [but] what's professional [here] might not be professional in a larger population ... simply because of the demographics and because everybody notices when somebody comes in the front door ... There are times when I think [people] have problems and maybe they don't come to talk about it because they want to keep it to themselves and they know that if they ... go [to counseling] everyone will know that they've gone to talk to the counselor.

Working within the cultural lens that confidentiality does not exist, clients reported talking freely about going to therapy with others and they expected that everyone else was talking about it, too. For example, the mother of the adolescent client in Case A explained:

The confidentiality thing is ... one of those things that you [therapists] keep to yourselves and [we], out here, just don't. I mean, [my children] tell people [about the counseling] and I talk to people at work. I mean, [there are] four or five [people I work with] that, you know, when [I'm talking about it], they overhear it all. And there's not a lot you're going to do about it. And so the confidentiality is ... it just doesn't exist out here ... You know, you talk about doctor confidentiality -if somebody gets told they have cancer, the whole town knows about it in five minutes, you know, and it's the same thing with [counseling]. 
Client willingness to disclose information about their attendance at therapy is obvious through an incident experienced by a therapist who traveled to the rural community to have a face-toface meeting with his clients. He explained that his adolescent client was waiting for him at the front door of the school. His client led him through the halls introducing him to friends. Teachers would come up to him and say "You must be Cody." He said, "Everyone knew who I was before I even got there."

While clients expressed discomfort at knowing that it was unavoidable to keep from public awareness the fact that they were attending therapy, none of the participants reported that receiving therapy through the BTH program compromised the confidentiality of the content of their sessions. In fact, rural participants readily acknowledged the professionalism of the therapists and their confidence that the content of their sessions was confidential. A surprising finding was that the therapist's "outsider" status appears to have helped this. Each client participant explained that because the therapist, as an outsider, was not wandering the streets of town, shopping where they shop, and living in their community, they could feel comfortable that the content of therapy was being kept confidential.

\section{Assertion 2: Technology Mediated Conversations Are Different Than Face-to-Face Conversations}

Technology exerts influence on the communication process. Without accommodations to this influence, communication can be disrupted and the resulting frustration can compromise the therapeutic relationship. This is important because the primary curative factor in psychotherapy - the "therapeutic alliance" - is dependent on client judgments of the helpfulness of their relationship with the therapist (Myer, et al., 2002). If the technology stands in the way of the development of a trustworthy helping relationship, then it could be assumed that treatment effectiveness would be compromised. However, the clients and therapists in this study reported that they were able to make necessary adjustments and accommodations to normal communication styles that compensated for the influence of the technology, thus preserving the therapeutic relationship.

Initial reluctance to participate in technology-mediated therapy was common for both client and therapist, due largely because of a lack of familiarity with the technology. With familiarity, respondents learned to make accommodations to the technology that allowed BTH to function similar in content and structure to face-to-face therapy. Below we will first discuss the issues present in dealing with the technology that could negatively influence the therapeutic alliance and then discuss the accommodations that were made to adjust to the technology, thus preserving the alliance.

Scheduling. Given the complexity of scheduling, great care had to be taken to ensure that the therapeutic relationship was not adversely affected by scheduling hassles. Therapists did this by assuming all responsibility for scheduling at both the clinic and enduser site. Interestingly, the male therapist identified "time spent in scheduling" as one of the most important issues to be considered for therapists. The female therapist described these challenges in scheduling in the following way.

[Working through so many people] is the most frustrating experience I have ever had of doing therapy. It's extremely difficult to get [so many] people to agree on one time ... me and my schedule, the client and their schedule, the school and their class schedule ... the connection in North Platte, $\ldots$ and the [telecommunications administrators], ... and then sometimes there might be other things in there. The room might be being used for a class, even though it's not a distance class [and] finding time that the FRC is available. There's a lot of coordination that has to happen.

Although attempts were made by therapists to contain all scheduling difficulties, clients were not completely shielded from experiencing scheduling hassles. For example, each client experienced at least one scheduled appointment where the technology room was not unlocked or otherwise prepared for him or her. This forced them to interact with high school staff in overcoming the problem. As a result of these difficulties, each of the clients resourcefully (and independent of the therapist) identified someone that they felt comfortable approaching to help them with scheduling. Although these were not people identified that the therapist or faculty were working with, they were persons that each of the clients felt they could trust with confidences. For example, the male therapist was surprised to learn that Client A resolved all his scheduling and access issues through the school janitor. Other clients said that they preferred to work through a secretary over those identified by the program as the contact people. Clients reported these personnel to be very professional and to be those whom they believed would not disclose any information about their attendance at therapy. 
Non-verbal communication. Research has consistently found nonverbal communication to be more instrumental in conveying a message than verbal communication. Assessment of non-verbal communication is especially important in psychotherapy. The BTH program designers were careful to select a telecommunications system that would allow the therapist and client to see one another from head-to-toe in an effort to maximize the ability to observe nonverbal behaviors. However, the fact that the therapist and client were not in the same room with one another restricted the non-verbal behavioral exchange. The mother of Client A described it as "talking to the TV" and explained, "If you had somebody who was really wanting to hide what they were feeling and what they were doing, I think it would be very easily done this way." Client B said, "I think that the technology impersonalizes [the relationship] to some degree ... There's sort of an unreal quality to it."

To compensate for the loss of non-verbal stimuli, the therapists instituted three accommodations. First, therapists reported that they were more deliberate and explicit in their non-verbal communication through the BTH medium than when conducting traditional face-to-face therapy. They both reasoned that because voice inflections, tonal changes, and body language cues are so important in conveying a therapeutic message, that deliberately exaggerating these behaviors compensated for the loss of these stimuli and facilitated the communication process. Observations of the video taped sessions also reflected that therapist non-verbal behavior appeared deliberate and exaggerated. However, clients appeared to draw from the exaggerated behavior the intended message to the point that the exaggerated behavior became a normal way of non-verbal communication that was even exhibited by clients.

Second, therapists reported asking a greater number of questions about their clients' non-verbal behavior. They said that these questions were needed to clarify the non-verbal cues of the client that are not as clear as they would be in face-to-face interactions. Once again, observations of the videotaped sessions revealed a substantial number of questions about client non-verbal behavior. Therapist participants reported that this technique facilitated therapy because non-verbal behavior was less likely to be misinterpreted.

Third, during the early portions of therapy, therapists offered to make a trip to the rural community to meet face-to-face with the client. Both of the therapists indicated that they felt that a face-toface meeting with the client was important in the joining process to compensate for non-verbal data lost through the telecommunications system. They explained that seeing their client face-to-face would provide them with a context in which to evaluate all future interactions. It was also believed that this would allow them to conduct a better assessment of client functioning. Although the invitation was made to each of the clients participating in the study, only Client A accepted the invitation. When asked why they did not want the face-to-face meeting, both Clients B and C referenced issues of privacy and confidentiality. Without prompting, Client B commented at the beginning of the interview- which was conducted in the rural county-that there were people in the community who make it their business to know what is going on with everyone else. She said that the fact that the interviewers were driving an unfamiliar car with urban county license plates would be cause for suspicion. Not surprisingly, she indicated that a personal interview with her therapist in her own community would not be desirable because it would make it even more difficult to keep private the fact that she was receiving therapy. However, the clients in Case A found the interview helpful. They said that it helped them to connect with the therapist. The mother in this case said that she noticed her son more willing to participate in therapy after the in-person meeting. The therapist confirmed this in reporting his observation that the adolescent client was more willing to disclose information after meeting face-to-face.

Signal delay. A delay in signal-common to most telecommunications options - was noted by both therapists and clients. Although the delay in signal is slight (less than one second), it is disruptive to normal communication patterns. For example, if a person expects to interject a statement such as "yes" or "u-hum" into the conversation, they can expect that by the time the talker hears the interjection they will have said up to an additional two to three words. So, communication does not move at as rapid a pace as it does in faceto-face conversations.

Each of the clients and therapists reported being able to adjust easily to the delay, but the adjustment depended on the degree to which they were comfortable with technology-mediated conversations. For example, Client A, who appeared to have the easiest time with the adjustment explained, "I've just gotten to the point where I wait a couple seconds before I respond. It's not all that difficult to get used to. Its not all that bad . . . it just took a little bit longer to say something and get a [response] back. After I got used to it, it 
was all right." While his mother reported experiencing greater difficulty adjusting, she too was able to make the adjustment during the first session. She said,

It was just too weird until you got used to that and used to how it was going to come across ... [I got used to it] kind of towards the end of the session ... you adjust to it ... When he got home that night [after the first session] I said, "How can you focus on that?" [He] says, "Oh mom, it's not that bad." Well it's just a getting used-to situation.

But, when asked if the signal delay interfered with the therapy, this same woman responded, "No, I don't think so . . . For some people it might, but I think that [the therapist] was so good with [us] . . . that it wasn't a problem that way."

The male therapist explained that the signal delay was most disruptive at the beginning portions of therapy when the therapist is trying to join with the client. He attributed this to a) the unfamiliarity of the clients with the technology and b) to the different pacing of communication that occurs when joining than when in the working stages of therapy. He explained that the delay was particularly disruptive for him when either he or the client "wanted to make a joke or smart-aleck comment" because these are so dependent on a particular timing in order to be successful. With practice, both therapists and clients learned to alter the pacing and timing of communication to compensate for the inconvenience of the signal delay.

Technical difficulties. There were instances in which either the equipment was not working properly or in which the connection between sites was lost or was not able to be established. These technical difficulties disrupted the flow of the sessions and in some cases prevented the session from occurring. Client $\mathrm{C}$ said:

We had some trouble with the technology. You know, there were a couple of times that it didn't just jive like it should, and we got started late, or ... I think there was one time we couldn't even get started. And, that didn't bother me. I figure that's a small price to pay for this convenience. And the setup, the delay in the speaking and the artificial TV type of thing, that didn't bother me at all either. .. I guess that I was more than willing to put up with any kind of technological inconvenience.
To accommodate these technological difficulties, therapists conducted some abbreviated sessions and some sessions by telephone. Although rescheduling was sometimes difficult, this was another accommodation. Both therapists explained that they learned to introduce the most significant therapeutic issues early in the session and to address those first. They said that their clients learned to do this as well to make the most of their time on-line. The chatting and social niceties-if they occurred in treatment-were reserved to the end of the session. The clients explained that they would just keep talking until the connection expired. When it did, they came to accept that this was the end of the session.

\section{DISCUSSION}

Through this research, the investigators identified two asser tions that describe both a) the barriers to accessing mental health care in rural communities (i.e. inaccessibility of mental health services and confidentiality) and $b$ ) the influences of technology on the communication process and the accommodations that need to be made to preserve the therapeutic relationship (i.e. scheduling, nonverbal communication, signal delay, technical difficulties).

The research provides some preliminary evidence to suggest that the BTH program can satisfactorily address the access to mental health care problem facing many rural communities. All rural participants indicated that they would choose the BTH program over all other options, and especially, over not receiving treatment at all. Traditional psychotherapeutic methods in which the therapist and clients meet face-to-face do not meet the logistic needs of most rural residents. Many residents live two to three hours away from therapeutic services and bear the financial burden of not only the session fee but also travel costs and work missed.

This research suggests that one of the primary issues to be addressed in delivering mental health care to rural communities through telecommunications is privacy and confidentiality. The participants explained that they believed that in rural communities everyone knows everyone's business, including whether they are getting mental health care. They said that they felt everyone would know regardless of where they would be receiving treatment. So, clients let people know themselves. It can be assumed that they did this to avoid others learning from rumors. However, at the same time, clients developed strategies to keep their attendance at ther- 
apy confidential. For example, Clients $\mathrm{B}$ and $\mathrm{C}$ declined the opportunity to meet face-to-face with their therapist because it would call attention to the fact that they were in therapy. Each of the clients identified someone at the school that they believed would be least likely to disclose their attendance or ask them about their attendance at therapy. In each case, those they identified were people different from those identified by the program faculty. This calls to attention the need to follow the lead of the client when making accommodations to the therapy in order to achieve success. It can reasonably be imagined that failure would have ensued had those working with the BTH program "required" that these accommodations not be made. Clients understand the context of their community much better than someone living hundreds of miles away and are in a better position to make appropriate accommodations.

Another noteworthy finding concerns the accommodations to the technology that must be made in order to preserve the therapeutic relationship. Although technology may negatively influence communication processes by restricting the amount of communication stimuli available to assist in the communication process, the results of this study suggest that these negative influences can be compensated for with appropriate accommodations to the technology and modes of communication. It is interesting to note that all participants made these accommodations naturally as they navigated the process of establishing and maintaining the therapeutic alliance. No protocol existed to direct therapists and clients in knowing what accommodations needed to be made.

This natural inclination to accommodate to limitations in the communication process may be an important consideration when investigating the role of technology in therapeutic conversations. It could be that the inaccessibility of services and the convenience of the BTH program motivates clients to forgive inconveniences associated with the technology. It could also be that people have different expectations of the communication process depending on the communication medium, thus, they might be more patient with these difficulties. For example, it may be easy to forgive a late start to therapy because everyone assumes the technology is to blame. Or, because some nonverbal information is not available, both therapists and clients may accommodate this lack of information by asking additional questions or providing additional information, thus, improving clarity. In many ways, this could actually benefit treatment. Therapists would be able to ask in a very natural way, about facial expressions, gestures, and other non-verbal communications. that they may not ask about when in the person's presence. Be- cause both clients and therapists are expecting that relationships will be more difficult to establish and maintain, they may put more work into the relationship.

Although the research did not compare BTH with face-to-face therapy, it provides suggestive evidence to the effectiveness of BTH treatment. Participants acknowledged that technology mediated treatment is different than face-to-face treatment. However, they also expressed satisfaction with BTH, and successful treatment progress and outcomes were reported. Simply, the fact that the first three cases seen through this medium can be considered treatment successes can certainly lead to speculation that effectiveness of treatment is not diminished over traditional treatments, but, additional research will be needed to answer this empirical question.

There are several notable limitations to this study. First, the study relied on a small purposeful sample. Consequently, these results are not generalizable. The study was designed as an exploratory investigation of a new program of treatment delivery. The results can be used to advance the field through the identification of issues that need to be considered as behavioral telehealth programs are developed and protocols are established. The results should be transferable to other program attempts.

Second, the study participants were all inexperienced. The clients were the first three clients to be treated with a new program of technology-mediated therapy. The therapists had no previous experience with behavioral telehealth. It is certainly possible that much different results would be derived from experienced BTH therapists operating out of a well-developed protocol of treatment delivery. It is also probable that as experience increases, the frequency of technological and other problems will decrease. However, this inexperience also provided rich data for the study. Clients and therapists were uncluttered with bias due to previous experience. So, rather than getting opinions from therapists about what should be done to accommodate the technology, the investigators had access to both therapist and client struggles as they attempted to make accommodations for the first time.

\section{REFERENCES}

Amundson, B. (2001). America's rural communities as crucibles for clinical reform: Establishing collaborative care teams in rural communities. Families, Systems, \& Health, 19(1), 13-23. 
Blumenthal, S. J., \& Kagen, J. (2002). The effects of socioeconomic status on health in rural and urban America. Journal of the American Medical Association, 287, 109-110.

Chalifoux, Z., Neese, J. B., Buckwlater, K. C., Litwak, E., \& Abraham, I. L. (1996). Mental health services for rural elderly: Innovative service strategies. Community Mental Health Journal, 32, 463-480.

Chavez, N. (1997). News from Washington, DC: Serving the underserved-our societal responsibility. Professional Psychology: Research and Practice, 28, 203-204.

Craft, J. L., \& Staudt, M. M. (1991). Reporting and founding of child neglect in urban and rural communities. Child Welfare, 70, 359-370.

Denzin, N. K. (1978). Sociological methods. New York: McGraw Hill.

Hoyt, R. D., Conger, R. D., Valde, J. G., \& Weihs, K. (1997). Psychological distress and help seeking in rural America. American Journal of Community Psychology, 23, 449-470.

Johnson, D. R., \& Booth, A. (1990). Rural economic decline and marital quality: A panel study of farm marriages. Family Relations, 39, 159-165.

Kim, K. J., Conger, R. D., Lorenz, F. O., \& Elder, G. H. Jr. (2001). Parent-adolescent reciprocity in negative affect and its relation to early adult social development. Developmental Psychology, 37, 775-790.

Miller, E. A. (2001). Telemedicine and doctor-patient communication: An analytical survey of the literature. Journal of Telemedicine and Telecare, 7, 1-17.

Moon, S. M., \& Trepper, T. S. (1996). Case study research. In D. H. Sprenkle \& S. M. Moon (Eds.), Research methods in family therapy (pp. 393-410). New York: Guilford Press.

Myer, B., Pilonis, P. A., Krupnick, J. L., Egan, M. K., Simmens, S. J., \& Stosky, S. M. (2002). Treatment expectancies, patient alliance and outcome: Further analyses from the National Institute of Mental Health Treatment of Depression Collaborative Research Program. Journal of Counseling and Clinical Psychology, 70, 1051-1055.

Nebraska Health \& Human Services (2002). Nebraska 2002 vital statistics report. Lincoln, NE: Department of Regulation \& Licensure and Department of Finance \& Support.

Nickelson, D. W. (1998). Telehealth and the evolving health care system: Strategic Opportunities for professional psychology. Professional Psychology: Research and Practice, 29, 527-535.

Roberts, L. W., Battaglia, J., Smithpeter, M., \& Epstein, R. S. (1999). An office on main street: Health care dilemmas in small communities. Hastings Center Reporter, 29(4) 28-37.

Rohland, B. M., Saleh, S. S., Rohrer, J. E., \& Romitti, P. A. (2000). Acceptability of telepsychiatry to a rural population. Psychiatric Services, 51, 672-674.

Strauss, A., \& Corbin, J. (1990). Basics of qualitative research. Newbury Park, CA: Sage Publications.

Wells, A. M., \& Durlak, J. A. (1998). Evaluation of indicated preventive intervention (secondary prevention) mental health programs for children and adolescents. American Journal of Community Psychology, 26, 775-802.

Yin, R. K. (1989). Case study research: Design and methods. Newbury Park, CA: Sage Publications. 